\title{
Transformation of structural defects in semiconductors under action of electromagnetic and magnetic fields causing resonant phenomena
}

\author{
G.V. Milenin', R.A. Red'ko ${ }^{1,2}$ \\ ${ }^{1}$ V. Lashkaryov Institute of Semiconductor Physics, National Academy of Sciences of Ukraine, \\ 41, prospect Nauky, 03028 Kyiv, Ukraine \\ ${ }^{2}$ State University of Telecommunications, 7, Solomenska str., 03680 Kyiv, Ukraine
}

\begin{abstract}
Possible mechanisms of transformation of defects in semiconductor structures under action of electromagnetic radiation in the microwave range and pulsed magnetic field have been analyzed. Electrical-resonance effects under nonthermal action of electromagnetic fields have been considered, namely: resonant detachment of dislocations and destruction of impurity complexes in semiconductor crystals, electrical-resonance transformation of defects in semiconductor crystals under action of weak pulsed magnetic fields; magnetic-resonance effects on defects in semiconductor crystals under action of weak magnetic and electromagnetic fields. It has been shown that alternative interaction mechanisms should be used to explain a large number of reliably established magnetically induced effects and phenomena associated with the nonthermal effects of microwave fields. There are two the most probable mechanisms: (i) spin-dependent reactions of paramagnetic defects in semiconductor crystals, as a result of which detachment and subsequent movement of dislocations in the field of internal stresses and (ii) resonant phenomena of various nature occur, which, generally, do not require high energies, and have been realized when the oscillation frequencies of the system and the external action coincide. A sharp increase in the amplitude of oscillations leads to detachment of dislocations and destruction of impurity complexes with subsequent movement and diffusion under action of a mosaic of internal mechanical stresses in the crystal. The principal physical identity of the influence of a weak magnetic field and nonthermal action of microwave radiation on a semiconductor material has been shown.
\end{abstract}

Keywords: microwave radiation, nonthermal action, weak magnetic field, resonant phenomena, spin reorientation.

doi: https://doi.org/10.15407/spqeo22.01.39

PACS 41.60.-m, 61.72.-y, 72.80.Ey, 73.50.Mx, 75.76.+j, 76.20.+q

Manuscript received 06.03.19; revised version received 25.03.19; accepted for publication 27.03.19; published online 30.03.19.

\section{Introduction}

The influence of both weak magnetic fields (it means that for these fields the relationship $\mu_{\mathrm{B}} B<<T$, where $\mu_{\mathrm{B}}$ is the Bohr magneton, $B$ - magnetic field induction, $k-$ Boltzmann constant, $T$ - absolute temperature, could be applied) and nonthermal effect of electromagnetic radiation of the microwave (MW) range on the state of defects in diamagnetic (semiconductor) crystals refer to the number of experimentally confirmed phenomena [15]. At the same time, this fact is nontrivial, since the energies of interaction of particles and defects in crystals under these fields are negligible as compared to the energy barriers that they need to overcome during their movement. Considering that the small effect leads to a significant physically observable response, it is quite reasonable to assume that these fields either act on the spin of the particles or cause resonant phenomena in the system of crystal defects.

The study of mechanisms providing these phenomena has both scientific and practical interest for the following reason. Improving the structural perfection of epitaxial films prepared from semiconductor structures $[6,7]$ and nanostructured objects $[8,9]$ through the nonthermal action of electromagnetic fields and treatments in weak magnetic fields is a promising direction. In turn, controlling the change in the microstructure of semiconductor materials is possible when understanding the mechanisms underlying the transformation processes of structural and impurity defects under action of the noted fields.

This article is aimed at the analysis of results obtained earlier, including the authors' works, and at development of new ideas. 


\section{Electrical-resonance effects under nonthermal action of electromagnetic fields}

\subsection{Resonant detachments of dislocations in semiconductor crystals}

In [10-12], it was shown that electrically charged dislocations fixed at the ends have their own (main) angular frequency of oscillations $\omega_{1}$ equal to:

$$
\omega_{1}=\frac{\pi}{L}\left(\frac{G}{\rho_{V}}\right)^{\frac{1}{2}} \text {, }
$$

where $G$ is the Poisson module, $\rho_{v}-$ bulk density of material, $L$ - dislocation length.

If the frequency of electromagnetic radiation $\omega_{e m}$ coincides with that of own dislocation oscillations, then the action of electrical component of the electromagnetic wave causes resonance phenomena. At the resonant frequency, the amplitude and, hence, the energy of oscillations at small damping increase sharply.

As soon as the oscillation energy exceeds the binding energy of the dislocations with the stoppers, then they become detached and begin to move under the action of internal mechanical stresses in the semiconductor crystal. This effect is characterized by a strong selectivity of the lengths of detachable dislocations to the frequency of electromagnetic radiation. In particular, for $n$-GaAs epitaxial structures at the frequency of electromagnetic radiation $v_{e m}=2.45 \cdot 10^{9} \mathrm{~Hz} \quad$ (the carrier frequency of the microwave generator used in the experiment) dislocations with the length $L=5.07 \cdot 10^{-7} \mathrm{~m}$ detach $[11$, $12]$.

At the same time, the resonator, in which the semiconductor structures were placed, is characterized by a wide spectrum of electromagnetic oscillations. On the other hand, the magnetron used as a microwave generator has the so-called pregeneration mode of operation, when in the spectrum of its output signal the electromagnetic oscillations in a rather wide frequency range are present. As a consequence, resonant detachment of a large number of dislocations with $L \leq 4.05 \cdot 10^{-6} \mathrm{~m}$ becomes possible [11].

\subsection{Resonant destruction of impurity complexes in semiconductors}

We shall analyze, in particular, destruction under the action of electromagnetic fields of defect complexes in $n$-GaAs epitaxial structures doped with tellurium. It is known that complexes (associates) "donor-acceptor" (impurity or impurity-defect complexes) are formed in $n$-GaAs crystals containing an uncontrolled copper impurity. Cations of tellurium $\left(\mathrm{Te}^{+}\right)$in the As sublattice are donors, and the acceptor is an impurity in the form of anions of copper $\left(\mathrm{Cu}^{-}, \mathrm{Cu}^{2-}\right)$ in the Ga sublattice [13].
Let us consider several possible scenarios for resonant destruction of impurity complexes $[11,12]$. Ionplasma oscillations of singly charged tellurium ions with an angular frequency $\omega_{p}$ are observed in the bulk of doped semiconductors outside the near-surface area of the space charge region $[11,14]$ :

$\omega_{P}=\left(\frac{e^{2} N}{\varepsilon \varepsilon_{0} M}\right)^{\frac{1}{2}}$,

where $N$ is the concentration of tellurium ions; $e-$ elementary electric charge (charge of electron); $\varepsilon-$ dielectric permittivity of semiconductor; $\varepsilon_{0}-$ electric constant of vacuum; $M$ is the mass of tellurium ion.

Thus, under the influence of the electrical component of electromagnetic radiation, the dynamics of tellurium ions is described by the equation of forced oscillations. If the frequency of the electromagnetic wave coincides with the plasma frequency of tellurium ions, then a resonance phenomenon will be observed. It is accompanied by a significant increase in the amplitude of oscillations of tellurium ions when their decay is small. At the same time copper ions make forced vibrations in the opposite direction with a small amplitude. The result will be destruction of impurity complexes with subsequent diffusion of copper. Calculations show that for $n$-GaAs epitaxial structures with $N=5 \cdot 10^{22} \mathrm{~m}^{-3}$ one can obtain $v_{P}=1.16 \cdot 10^{9} \mathrm{~Hz}\left(\omega_{P}=2 \pi v\right)[11]$.

Next, we shall consider the following possible scenario of destruction of impurity complexes in the near-surface area of the space charge in the semiconductor crystal. Suppose these associates are united in an electrically neutral cluster. Then, this cluster in the near-surface area of a semiconductor, where there is depleting band bends, is a nanostructural similarity of an ionic crystal [11, 12].

Behavior of tellurium and copper ions under the action of the electrical component of the electromagnetic field obeys the equation of forced oscillations. The ionplasma oscillation frequency in the case of singly charged copper ions, if taking into account that the concentrations of tellurium and copper ions in these electrical neutral clusters are the same and equal $N$, obeys the following relation $[11,12,15]$ :

$\omega_{P 1}=\left(\frac{e^{2} N}{\varepsilon \varepsilon_{0} \mu_{1}}\right)^{\frac{1}{2}}$,

where $\mu_{1}$ is the reduced mass of a pair of ions [15]:

$\mu_{1}=\frac{M_{1} M_{2}}{M_{1}+M_{2}}$,

here, $M_{1}$ is the mass of tellurium ion, $M_{2}$ is the mass of copper ion. 
The coincidence of the frequency of an electromagnetic wave with an ion-plasma frequency will be accompanied by a resonant increase in the amplitude of oscillations of ions and by destruction of impurity complexes and, consequently, of clusters consisting of them with subsequent diffusion of decomposition products.

If the complexes, from which an electrical neutral cluster is formed, are created by doubly charged copper ions, then one can formally consider two tellurium ions included into this associate as one ion with the charge $+2 e$ and mass $2 M_{1}$. The corresponding expression for the frequency of ion-plasma oscillations follows from (3) and (4) by replacing $e$ with $2 e$ and $N$ with $N / 2$, and has the form:

$\omega_{P 2}=\left(\frac{2 e^{2} N}{\varepsilon \varepsilon_{0} \mu_{2}}\right)^{\frac{1}{2}}$,

where

$\mu_{2}=\frac{2 M_{1} M_{2}}{2 M_{1}+M_{2}}$

The calculations performed [11] for $n$-GaAs epitaxial structures give $v_{P 1}=2.01 \cdot 10^{9} \mathrm{~Hz}$ and $v_{P 2}=2.6 \cdot 10^{9} \mathrm{~Hz}$. The obtained values are close to the above carrier frequency of the microwave generator.

As already noted, since electromagnetic oscillations in a wide frequency range take place in the resonator, resonance destruction of the impurity-defect complexes analyzed together with resonant detachment of dislocations, lengths of which satisfy the above relation, becomes possible. The subsequent displacement of dislocations and ions leads, in particular, to long-term non-monotonic changes in the intensity of the photoluminescence bands after microwave treatments [10-12, 16-18].

\section{Electrical-resonance transformation of defects in semiconductor crystals under the action of weak pulsed magnetic fields}

When a semiconductor crystal is placed into a uniform magnetic field with the induction $B$, conduction electrons, if their mass is a scalar value, begin to move along spiral trajectories, i.e. helical lines, axis of which coincides with the magnetic induction vector. It means that a charged particle simultaneously participates in two motions: under the action of the Lorentz force, it rotates in a circle with the velocity $v_{\perp}$ (component of the electron thermal velocity that is perpendicular to the magnetic induction vector), and also moves uniformly and rectilinear with the velocity $v_{\|}$(component of the electron thermal velocity that is parallel to the vector of magnetic induction). Understandably, if $v_{\|}=0$, then electron only rotates around a circle.
The angular velocity of rotation of electron, in other words the cyclotron frequency $\omega_{B}$, is equal to [19]:

$\omega_{B}=\frac{e B}{m_{n}}$,

where $m_{n}$ is the effective mass of electron in a semiconductor crystal.

Since during rotation electron undergoes acceleration that is constantly in magnitude and is directed perpendicular to the velocity vector, it is a source of electromagnetic wave radiation at a frequency $\omega_{B}$ [19].

The power of electromagnetic waves $W$ radiated by electrons per unit volume of a semiconductor crystal is equal to [20]:

$W=\frac{n e^{2} \omega_{B}^{2} v_{T}^{2}}{12 \pi \varepsilon \varepsilon_{0} c^{3}}$,

where $n$ is the concentration of free electrons in a semiconductor; $v_{T}=\left(3 k T / m_{n}\right)^{1 / 2}-$ mean square thermal velocity of electrons; $c$ - speed of light.

In particular, for $n$-GaAs epitaxial structures doped with tellurium with the concentration of free electrons equal to $5 \cdot 10^{22} \mathrm{~m}^{-3}$ in the magnetic field with $B=0.06 \mathrm{~T}$ at $T=300 \mathrm{~K}$, we have $v_{B}=2.7 \cdot 10^{10} \mathrm{~Hz}$ and $W=67 \mathrm{~W} \cdot \mathrm{m}^{-3}[21]$.

When creating a pulsed magnetic field by rotating a semiconductor crystal in the field of a magnet, the induction in the gap of which is $B=0.06 \mathrm{~T}$, the epitaxial structure moves through the region of the edge magnetic fields with the induction that is orders of magnitude less. Accordingly, the cyclotron frequency will also be lower by orders of magnitude. Consequently, in a semiconductor crystal, electromagnetic waves of a wide frequency range will be generated.

Thus, the mechanisms of transformation of the defect structure in epitaxial semiconductor films exposed to pulsed magnetic fields are the same as under the nonthermal effect of electromagnetic radiation of a wide frequency range [21]. As noted above, transformation of the subsystem of defects under the influence of electromagnetic radiation is caused by the detachment of dislocations, as well as destruction of impurity-defect complexes due to resonance phenomena.

The notion of common mechanisms of exposure to the electromagnetic field of the microwave range and weak pulsed magnetic field is confirmed both by a rather good agreement of the values of the diffusion coefficients for migrating impurities obtained in the authors' works for the epitaxial structures of GaAs and $\mathrm{GaN}$ after above treatments $[11,12,21]$ and experimental data $[5,22]$.

In [5], the importance of treatments with precisely pulsed magnetic field was emphasized, as well as the threshold nature and phenomenon of saturation on $B$ of changes induced by a magnetic field were also detected. This feature can be justified by the fact that the resonant frequencies of defect oscillations lie in a certain range. 
Then the frequencies of electromagnetic radiation must also lie in this range.

Therefore, in accordance with (7), both the threshold in $B$ nature of the effects induced by the magnetic field and the saturation effect should be observed. Finally, it was noted in [5] that at low temperatures the corresponding effects were poorly pronounced or completely absent. These phenomena can be explained by the fact that with decreasing temperature the concentration of charge carriers and their thermal velocities decrease, and consequently, according to (8), the power of cyclotron radiation also decreases. As a result, the amplitude of the electrical component of electromagnetic radiation decreases.

In turn, the amplitude of forced oscillations of defects is directly proportional to the amplitude of the disturbing force. Presented in [22] are the data on the change in the structural perfection of the near-surface layers in $\mathrm{A}^{\mathrm{III}} \mathrm{B}^{\mathrm{V}}$ semiconductor compounds after action of magnetic field pulses, indicating generation, transformation, and destruction of clusters of Frenkel defects. These changes are increased in the series of GaAs-InAs-InSb. This feature, apparently, is caused by the fact that in the noted series the effective masses of free charge carriers decrease.

Accordingly, taking into account (7) and (8), both the frequency $\omega_{B}$ increases, consequently, the range of the frequency spectrum expands, and the power of electromagnetic radiation increases, too. Both of these circumstances lead to an increase in changes in the structural perfection of the near-surface layers of semiconductor compounds after the action of magnetic field pulses.

\section{Magnetic-resonance effect on defects in semiconductor crystals under the action of weak magnetic fields}

The corresponding mechanisms are well studied for diamagnetic dielectric crystals with paramagnetic impurities. The change in the state of defects in these crystals under the action of a weak magnetic field, as well as in crossed magnetic and electromagnetic fields (in this case, crossed fields are those in which the magnetic induction vectors of the fields are mutually perpendicular, in other words, the magnetic induction vector of the constant field is perpendicular to the polarization plane of magnetic component of an electromagnetic wave) is caused by singlet-triplet transitions in the radical pair defect-stopper, leading to detachment of dislocations from stopper [1-3, 23].

A weak magnetic field removes the spin forbiddance, that is, it provides spin conversion, which, in turn, leads to a change in the rate of reactions [1-3, 23]. Accounting the same notions about spin-dependent reactions, magnetoplastic effects in semiconductor crystals are also explained $[1,3,24-26]$. In semiconductor materials, paramagnetic centers (defects) are dangling bonds carrying unpaired electrons in the

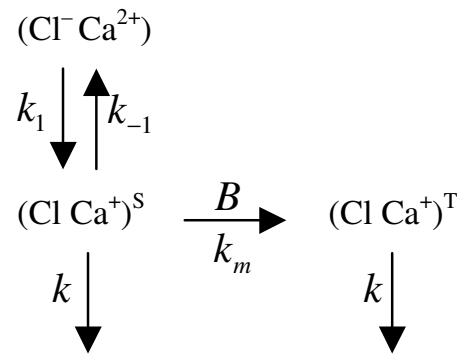

Destruction of pairs (detachment of dislocations)

Fig. 1. Transformation of a pair in the field with the magnetic induction $B\left(k_{1}, k_{-1}\right.$ are the constants of rate of generation and annihilation of the spin nanoreactor, respectively; $k_{m}, k-$ constants of rate for the spin conversion and dislocation detachment, respectively) [3].

core of dislocations [27], as well as oxygen atoms that often act as stoppers [3, 24-26]. In addition, donor and acceptor ions can be magnetically sensitive stoppers $[24,26]$.

Let us consider in more detail the mechanisms of spin-dependent reactions in dielectric and semiconductor crystals. The physics of this phenomenon was analyzed in detail in $[1-3,23]$ by using the example of the $\mathrm{NaCl}$ ionic crystal with the diamagnetic impurity $\mathrm{Ca}^{2+}$.

The stopper-dislocation system at the atomic-level can be represented as a pair $\left(\mathrm{Cl}^{-} \mathrm{Ca}^{2+}\right)$, and $\mathrm{Cl}^{-}$denotes an ionic dislocation element, interacting with $\mathrm{Ca}^{2+}$ stopper by means of Coulomb force. Evolution of this pair in a magnetic field is shown in Fig. 1 [3, 23]. The constituent stages of this transformation are generation of a spin nanoreactor in the singlet state $\mathrm{S}$ and its conversion into the triplet state $\mathrm{T}$ under the action of a magnetic field $[3,23]$.

A spin (spin-selective, magnetically sensitive) nanoreactor means two unpaired electrons, one of them is at a stopper and another - at a dislocation, and generation of a spin reactor is the transfer of electron from a dislocation to a stopper, as a result of which two unpaired electrons appear. In a spin-selective system formed by a pair of paramagnetic particles, the magnetic field controls spin conversion and removes the forbiddance on the occurrence of certain reactions.

As already noted, the spin nanoreactor can be in the following states: singlet, when the total spin of two electrons is zero, and triplet, when the total spin equals unity. In the singlet pair $\left(\mathrm{Cl} \mathrm{Ca}^{+}\right)^{\mathrm{S}}$ formed by transfer of electron from the dislocation to the stopper without magnetic field, Coulomb interaction is turned off, and, therefore, the dislocation acquires the ability to move under the influence of a mosaic of internal stresses in crystals. However, the probability of this event is small, because of the lifetime of singlet spin state is small. The latter circumstance is due to the fact that the reverse electron transfer restores the stopper-dislocation system $[3,23]$. 
The magnetic field transforms a singlet pair into the triplet one, at that spin conversion $\mathrm{S}_{-} \mathrm{T}_{0}$ occurs as a result of dephasing two spins, precessing with Zeeman (Larmor) frequencies $g_{1} \mu_{B} B / \hbar$ and $g_{2} \mu_{B} B / \hbar$, where $g_{1}$, $g_{2}$ are the $g$-factors of electron, $\hbar$ is Planck's constant. The rate of dephasing is proportional to $\Delta g$, at that $\Delta g=g_{1}-g_{2}$ is the difference of $g$-factors [1-3, 23]. Energy levels, spin states of nanoreactor (in magnetic field, the triple state splits into three substates: $T_{0}, T_{+}$and $\mathrm{T}_{-}$, in which the projections of the electron spin are equal to $0,+1$ and -1 , respectively) and the transition scheme $\mathrm{S}^{-\mathrm{T}_{0}}$ are shown in Fig. 2 [3].

In the triplet state, the Coulomb interaction is turned off like to that in the pair $\left(\mathrm{Cl} \mathrm{Ca}^{+}\right)^{\mathrm{S}}$, but the lifetime of the spin nanoreactor is much longer, since reverse electron transfer is forbidden along the spin, which, in turn, increases the probability of dislocation detachment [3, 23] leading to an experimentally observed increase in their runs $[1,2]$. This probability is higher, the shorter the destruction time of the triplet pair (removal of reaction products) as compared with the reverse transition time (reverse spin conversion) $\mathrm{T}_{0}-\mathrm{S}[2,23]$.

(a)

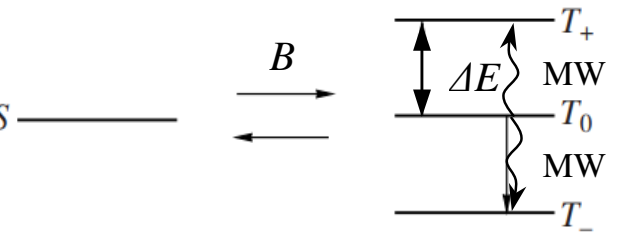

(b)
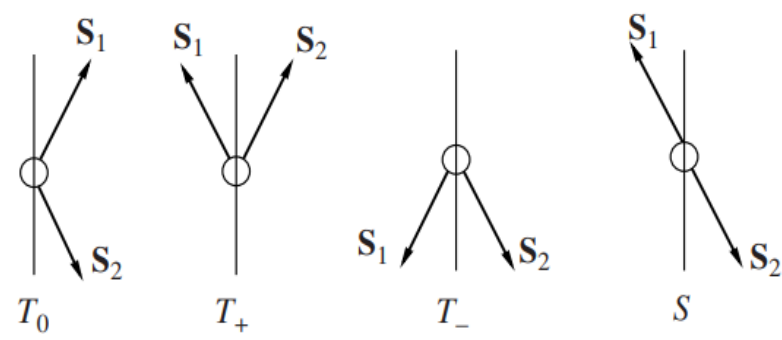

(c)

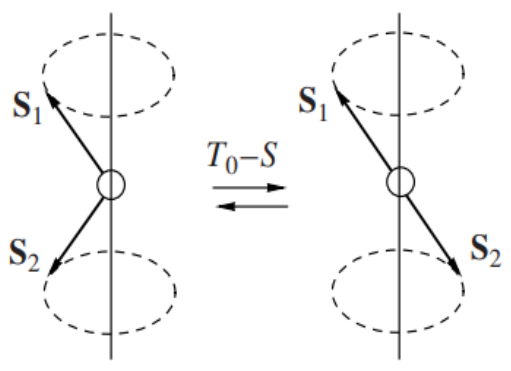

Fig. 2. The energy levels of a double-spin nanoreactor in the singlet $S$ and triplet $\left(T_{0}, T_{ \pm}\right)$states (a), the relative position of the spins in these states (b), and the scheme of S-T transition as spin dephasing (c); $\Delta E$ is the Zeeman splitting of levels; MW microwave field; the arrows denote the electron spins $S_{1}$ and $S_{2}$ for each of the nanoreactor components [3].
The combined action of crossed constant magnetic and microwave fields on the crystal induces, in addition to $\mathrm{S}-\mathrm{T}_{0}$ spin transitions causes also transitions between $\mathrm{T}_{ \pm}$ and $\mathrm{T}_{0}$ levels splitted by Zeeman interaction (Fig. 2) [1, 3]. These transitions become possible, when the condition of electron paramagnetic resonance is fulfilled $[1,3]$ :

$\Delta E=\hbar \omega_{e m}$,

moreover, the splitting of levels is:

$\Delta E=g \mu_{B} B$,

where $\omega_{e m}$ is the angular frequency of the microwave radiation.

The effects caused by such microwave fields are called magnetic resonance. Thus, the electromagnetic radiation in the microwave range, the frequency of which satisfies the condition (9), reorients the electron spins due to transitions from the state $T_{0}$ to the states $T_{ \pm}$. The $T_{0}$ level is partially emptied in this case. In turn, the population of the $T_{0}$ level occurs due to the $S-T_{0}$ transitions. It is noteworthy that direct transitions from the $\mathrm{T}_{ \pm}$states to the $\mathrm{S}$ one and backward are completely forbidden. The latter circumstance is the cause of an additional increase in the lifetime of the spin nanoreactor in the triplet state [1-3]. As a result, in ionic crystals the probability of dislocation detachment increases, and their run increases additionally [1-3].

It is important to emphasize that a significant difference between semiconductor crystals and dielectric crystals is the presence of free charge carriers in the semiconductor crystals. The dynamics of the subsystem of free charge carriers in a magnetic field may be the reason of these magnetic resonance effects that will be observed in the absence of an external electromagnetic microwave field.

Indeed, it was noted in Section 3 that the conduction electrons of a semiconductor crystal placed into magnetic field serve as a source of electromagnetic waves with the cyclotron frequency $\omega_{B}$. Substituting its expression from (10) into (9) instead of $\Delta E$ and replacing $\omega_{e m}$ with (7) as well as taking into account that the Bohr magnetron is $\mu_{\mathrm{B}}=e \hbar / 2 m_{0} \quad\left(m_{0}\right.$ is the mass of electron), it can be concluded that the value of the Zeeman splitting $\Delta E$ is equal to the cyclotron radiation quantum $\hbar \omega_{B}$, if the following relationship holds:

$g \frac{m_{n}}{2 m_{0}}=1$.

If the magnetic moment of a paramagnetic particle is caused by only the electron spin, then the $g$-factor equals 2 . However, even for free electron $g \approx 2$ due to quantum-electrodynamic effects. On the other hand, since in semiconductor crystals the strong electric fields of surrounding atoms act on paramagnetic particles, the 
$g$-factor of electron can take the values that are both less and more than two [26], including those that turn (11) into identity.

In addition, the following important circumstance must be taken into account. The spectral lines of cyclotron radiation of electrons in a semiconductor crystal are not infinitely narrow. Indeed, charged carriers, when moving under the action of magnetic field, experience acts of scattering by impurity ions and atoms, dislocations, acoustic and optical phonons. This leads to the broadening the cyclotron radiation line, and, consequently, to the presence of frequencies in the spectrum of the latter, which satisfy the condition of electron paramagnetic resonance (9).

As noted above, another condition for observing this resonance is the mutual perpendicularity of the magnetic induction vector describing the constant magnetic field to the polarization plane of the magnetic component of an electromagnetic wave. In turn, it is known that cyclotron radiation propagating in the direction normal to the plane of electron rotation has circular polarization [19].

It means that for electrons that have no thermal velocity component parallel to the induction vector of the constant magnetic field $\left(v_{\|}=0\right)$, that is, rotating along the circle in a plane perpendicular to this vector, this requirement is satisfied.

Thus, in semiconductor crystals, the observed magnetoplastic effects can be caused by the reorientation of electron spins due to subsequent transition of the paramagnetic pair first from the state $S$ to the state $T_{0}$ under the action of the constant magnetic field, and then from the state $T_{0}$ to state $T_{ \pm}$under the action of charge carrier cyclotron radiation.

In general, in semiconductors, transformation of defects, i.e. the detachment of dislocations from the stoppers, is a consequence of the magnetic resonance effect. This differs from the situation that takes place in dielectric crystals, where $\mathrm{S}-\mathrm{T}_{0}$ spin transitions are stimulated by the constant magnetic field, and the transitions $\mathrm{T}_{0}-\mathrm{T}_{ \pm}$are caused by the combined action of the constant magnetic and external microwave fields.

Consider the use of a spin nanoreactor to explain the magnetoplastic effects observed in silicon crystals [5, 19-21]. The process of detaching dislocations in the constant magnetic field, when the stoppers are oxygen atoms, can proceed as follows. Fixation of a dislocation occurs as a result of the attachment of an oxygen atom by a dangling $\mathrm{Si}$ bond in the dislocation core with formation of a Si-O fragment. Under the action of thermal fluctuations, this fragment can destruct with forming singlet pair consisting of $\mathrm{Si}$ and $\mathrm{O}$ paramagnetic particles, which is a spin nanoreactor.

As a result, the stopper ceases to hold the dislocation that under the action of internal mechanical stresses acquires the ability to move. However, the probability of such a detachment scenario for the dislocation is small due to the reverse process of restoring the $\mathrm{Si}-\mathrm{O}$ bond. At the same time, the constant magnetic field and cyclotron radiation of free charge carriers transit the singlet pair into the triplet one:

$(\mathrm{Si}-\mathrm{O}) \leftrightarrow(\mathrm{OSi})^{\mathrm{S}} \stackrel{B, \hbar \omega_{B}}{\longrightarrow}(\mathrm{OSi})^{\mathrm{T}}$

which makes it impossible, due to spin forbiddances, to restore the initial $\mathrm{Si}-\mathrm{O}$ fragment, and, therefore, initiates the process of pair destruction, that is, detaching and subsequent displacement of the dislocation.

In a very similar way, it is possible to analyze the dynamics of dislocations, when the ions of donors, in particular phosphorus, act as paramagnetic stoppers. In this case, fixation of dislocations is caused by formation of the $\mathrm{P}^{+}-\mathrm{Si}$ fragment. Like to the previous case, in a magnetic field its destruction takes place according to the scheme:

$\left(\mathrm{P}^{+}-\mathrm{Si}\right) \leftrightarrow\left(\mathrm{P}^{+} \mathrm{Si}\right)^{\mathrm{S}} \stackrel{B, \hbar \omega_{B}}{\longrightarrow}\left(\mathrm{P}^{+} \mathrm{Si}\right)^{\mathrm{T}}$

with subsequent detachment and movement of dislocations.

However, in semiconductor crystals, in contrast to ionic crystals, the combined action of the constant magnetic and external microwave fields leads to anchoring of dislocations, which experimentally manifests itself in a decrease in their runs relative to those that occurred under the action of only the constant magnetic field $[1,26]$.

So, in single crystals of silicon under the action of a mechanical load in the absence of microwave field, the run of dislocations increases with increasing the magnetic induction [26]. With the joint action of the fields, it was characterized by a wide minimum at the value of magnetic induction satisfying the condition of paramagnetic resonance [26].

The fact that the values of the Zeeman frequency of electron precession coincide with the cyclotron radiation frequencies allows us to explain also the effect of attachment of dislocations. Since the frequency of the external electromagnetic field is equal to the listed frequencies, then observed in semiconductor there will be the phenomenon of resonant absorption of electromagnetic energy by charged carriers - cyclotron resonance. As a result, electrons move along unwinding spirals and the power absorbed by them increases.

The growth of the latter is limited by scattering of charge carriers by lattice defects and phonons. The transfer of electron energy to the lattice leads to an increase in the mobility of defects that can act as stoppers and to movement of them towards dislocations. Precisely, the increase in the number of stoppers near dislocations causes the effect of the prevalence of their attachment over detachment under the combined action of constant magnetic and microwave fields. 


\section{Magnetic resonance changes in the state of defects in semiconductors stimulated by the action of electromagnetic fields}

The magnetic component of an electromagnetic wave can cause spin-dependent reactions in paramagnetic defects of semiconductor crystals according to the scenario described in the previous section. However, a necessary condition for the change in multiplicity, i.e., the transition from the singlet state to the triplet one is as follows: the frequency of the transition from the state $S$ to $\mathrm{T}_{0}$ and vice versa $\mathrm{v}_{\mathrm{S}-\mathrm{T}_{0}}$ should be much higher than the frequency of electromagnetic radiation $\mathrm{v}_{e m}$, that is $\mathrm{v}_{\mathrm{S}-\mathrm{T}_{0}}>>\mathrm{v}_{e m}$. In this case, the magnetic component of the electromagnetic field can be considered as quasi-static. The characteristic frequencies of spin conversion $\left(\mathrm{S}-\mathrm{T}_{0}\right.$ transitions) are $10^{7}-10^{9} \mathrm{~Hz}[2,23]$.

Accordingly, the frequencies of the electromagnetic fields should not exceed the values $v_{e m}=10^{6}-10^{8} \mathrm{~Hz}$. Thus, this mechanism of transformation of defects cannot take place in semiconductor crystals under the action of microwave radiation on them. Therefore, for a given external action, to interpret the corresponding results, it is necessary to involve notions about the resonant behavior of dislocations and impurity-defect complexes, which were discussed in Section 2.

\section{Conclusion}

Thus, the basis for evolution of the defect subsystem in semiconductor structures under the nonthermal effects of electromagnetic radiation in the microwave range is electrical resonance phenomenon caused by coincidence of the frequencies of the latter with the own and ionplasma frequencies inherent to oscillations of dislocations and ions in impurity-defect complexes. At the resonant frequency, due to a sharp increase in the amplitude of these oscillations, detachment and subsequent displacement of dislocations occur under the action of a mosaic of internal mechanical stresses of the crystal, as well as destruction of impurity associates with diffusion of degradation products.

Transformation of the defect structure in epitaxial semiconductor films under the action of pulsed magnetic fields can occur by two scenarios. One of them supposes the spin-dependent reactions in paramagnetic defects of semiconductor crystals. In this case, detachment and subsequent displacement of dislocations are stimulated by magnetic resonance electron transitions in the paramagnetic pair of defect-stopper to the triplet levels splitted by Zeeman interaction. These transitions become possible under equality of frequencies of cyclotron radiation of free charge carriers in the crystal and Zeeman precession of electron.

Laid into the basis of another scenario is the notion about the identity of the effects of magnetic and electromagnetic fields on the semiconductor crystal. Indeed, in a semiconductor structure under the action of a pulsed magnetic field, cyclotron radiation with a wide frequency range is generated. The coincidence of these frequencies with the frequencies of oscillations of defects leads to electrical-resonance effects. The notion of a total electrical-resonance mechanism of the action of electromagnetic radiation in the microwave range and a pulsed magnetic field is in good agreement with experimental data.

\section{References}

1. Morgunov R.B. Spin micromechanics in the physics of plasticity. Phys. Usp. 2004. 47. P. 125-147.

2. Golovin Yu.I. Magnetoplastic effect in solids (Review). Physics of the Solid State. 2004. 46, No 5. P. 789-824.

3. Buchachenko A.L. Magnetoplasticity of diamagnetic crystals in microwave fields. Journal of Experimental and Theoretical Physics, 2007. 105(3), P. 593-598. DOI: 10.1134/S1063776107090166.

4. Belyavsky V.I., Levin M.N. Spin effects in defect reactions. Phys. Rev. B. 2004. 70, No 10. P. 104101-1-104101-8.

5. Levin M.N., Zon B.A. The effect of pulsed magnetic fields on $\mathrm{Cz}-\mathrm{Si}$ crystals. Journal of Experimental and Theoretical Physics. 1997. 84, No 4. P. 760-773.

6. Levin M.N., Tatarintsev A.V., Kostsova O.A., Kostsov A.M. Activation of a semiconductor surface by a pulsed magnetic field. Tech. Phys. 2003. 48, No 10. P. 1304-1306.

7. Levin M.N., Semenova G.V., Sushkova T.P., Dolgopolova E.A., Postnikov V.V. The effect of pulsed magnetic fields on the real structure of indium arsenide crystals. Tech. Phys. Lett. 2002. 28, No 10. P. 818-820.

8. Rodríguez-Castañeda C.A., Moreno-Romero P.M., Martínez-Alonso C., and $\mathrm{Hu} \mathrm{H}$. Microwave synthesized monodisperse $\mathrm{CdS}$ spheres of different size and color for solar cell applications. $J$. Nanomater. 2015. 2015. Article ID 424635. 10 p.

9. Gerbec J.A., Magana D., Washington A., and Strouse G.F. Microwave-enhanced reaction rates for nanoparticle synthesis. J. Amer. Chem. Soc. 2005. 127, No 45. P. 15791-15800.

10. Ermolovich I.B., Milenin G.V., Milenin V.V., Konakova R.V., Red'ko R.A., Modification of the defect structure in binary semiconductors under action of microwave radiation. Techn. Phys. 2007. 52, No 9. P. 1173-1177.

11. Milenin G.V., Red'ko R.A. Physical mechanisms and models of the long-term transformations in radiative recombination observed in $n$-GaAs under microwave irradiation. Semiconductor Physics, Quantum Electronics \& Optoelectronics. 2016. 19, No 1. P. 14-22.

12. Red'ko R.A., Milenin G.V., Milenin V.V. Mechanisms and possibilities of defect reorganization in III-V compounds due to the nonthermal microwave radiation treatment. $J$. Luminesc. 2017. 192. P. 1295-1299. 
13. Masterov V.F., Samorukov B.E., Deep centres in $\mathrm{A}^{\mathrm{III}} \mathrm{B}^{\mathrm{V}}$ compounds (Review). Fizika $i$ tekhnika poluprovodnikov. 1978. 12, No 4. P. 625-652 (in Russian).

14. Ashcroft N., Mermin D. Solid State Physics. Thomson Press (India) Ltd, 2003.

15. Smith R.A. Semiconductors. Cambridge, New York, Cambridge University Press, 1978.

16. Zayats N.S., Konakova R.V., Milenin V.V., Milenin G.V., Red'ko R.A., Red'ko S.N. Microwaveradiation induced structural transformations in homo- and heterogeneous GaAs-based systems. Techn. Phys. 2015. 60, No 3. P. 432-436.

17. Ermolovich I.B., Milenin V.V., Konakova R.V., Red'ko R.A. Influence of microwave irradiation on radiative recombination in $\mathrm{A}^{3} \mathrm{~B}^{5}$ compounds. Fizika $i$ khimiya obrabotki materialov, 2006. № 5. P. 1218 (in Russian).

18. Milenin V.V., Red'ko R.A., Red'ko S.N. Effect of microwave irradiation on radiative recombination in GaAs. Radioelectronics and communications systems. 2006. 8, No 9. P. 77-80.

19. Bekefi G. Radiation Processes in Plasmas. Wiley, New York-London-Sydney, 1966.

20. Milenin G.V., Milenin V.V., Red'ko R.A. Cyclotron radiation of semiconductor crystals. Semiconductor Physics, Quantum Electronics \& Optoelectronics. 2018. 21, No 1. P. 54-57.

21. Milenin G.V., Red'ko R.A. Physical mechanisms and models of long-term transformations of radiative recombination in $n$-GaAs due to the magnetic field treatments. Semiconductor Physics, Quantum Electronics \& Optoelectronics. 2016. 19, No 3. P. 279-284.

22. Davydov V.N., Loskutov E.A., Naiden E.P. Late structural changes in semiconductors, stimulated by magnetic field. Semiconductors. 23(9), p.1596 1600 (1989), in Russian.

23. Buchachenko A.L. Effect of magnetic field on mechanics of non-magnetic crystals: The nature of magnetoplasticity. Journal of Experimental and Theoretical Physics, 102(5), p. 795-798 (2006).

24. Skvortsov, A.A., Orlov, A.M. \& Gonchar, L.I. The effect of a weak magnetic field on the mobility of dislocations in silicon. Journal of Experimental and Theoretical Physics. 93(1), p. 117-120 (2001).

25. Badylevich, M.V., Iunin, Y.L., Kveder, V.V. et al. Effect of a magnetic field on the starting stress and mobility of individual dislocations in silicon. Journal of Experimental and Theoretical Physics, 97(3), p. 601-605 (2003).

26. Ossipyan, Y.A., Morgunov, R.B., Baskakov, A.A. et al. Magnetoresonant hardening of silicon single crystals. Journal of Experimental and Theoretical Physics Letters. 79(3), p. 126-130 (2004).

27. Ossipyan Y.A., Bredikhin S.I., Kveder V.V. et al. Electronic Properties of Dislocations in Semiconductors. Moscow, Editorial URSS, 2000 (in Russian).

\section{Authors and CV}

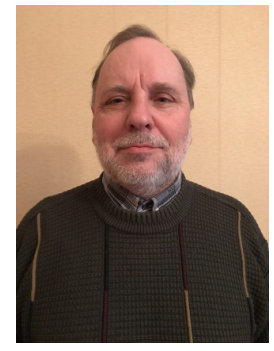

Milenin G.V.: PhD "Solid-State Physics", Senior Researcher.

V. Lashkaryov Institute of Semiconductor Physics, National Academy of Sciences of Ukraine E-mail: milenin.gv@gmail.com

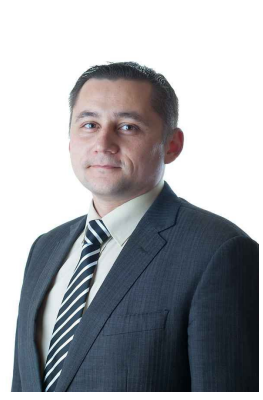

Red'ko R.A.: PhD "Solid-State Physics", Senior Researcher, Associate Professor.

V. Lashkaryov Institute of Semiconductor Physics, National Academy of Sciences of Ukraine E-mail: redko.rom@gmail.com 